\title{
THE TAYLOR SERIES FOR BANDLIMITED SIGNALS
}

\author{
M. A. HERNANDEZ VERON ${ }^{1}$
}

(Received 28 April 1992; revised 27 November 1992)

\begin{abstract}
We study a special type of infinite product, called an infinite product of Cardano type, and we obtain its Taylor series. We prove that Hadamard's factorization of bandlimited signals is given by an infinite product of Cardano type, and apply our results to obtain the Taylor series for bandlimited signals.
\end{abstract}

\section{Introduction}

Let $Q$ be a polynomial of degree $q$, normalized by $Q(0)=1$ and with roots $\left[w_{n}\right]_{n=1, \ldots, q}$. Cardano's formulae allow us to represent $Q$ in the form

$$
Q(z)=\prod_{n=1}^{q}\left(1-\frac{z}{w_{n}}\right)=\sum_{k=0}^{q}(-1)^{k} D_{k} z^{k},
$$

where $D_{0}=1$ and $D_{k}=\sum_{1 \leq i_{1}<\ldots<i_{k} \leq q} w_{i_{1}}^{-1} \ldots w_{i_{k}}^{-1}$ for $1 \leq k \leq q$.

The main purpose of this paper is to extend this result to bandlimited signals. Let $f$ be a bandlimited signal which has been normalized by $f(0)=1$. We investigate when we can write

$$
f(z)=\prod_{n \geq 1}\left(1-\frac{z}{w_{n}}\right)=\sum_{k \geq 0}(-1)^{k} D_{k} z^{k},
$$

where $\Lambda=\left\{w_{n}: n \geq 1\right\}$ is the set of zeros of the function $f$, and where $D_{0}=1$ and for $k \geq 1$ :

$$
D_{k}=\lim _{n} \sum_{1 \leq i_{1}<\ldots<i_{k} \leq n} w_{i_{1}}^{-1} \ldots w_{i_{k}}^{-1} .
$$

Notice that $\Lambda$ is a countable set because in our conditions $f$ is analytic in the entire plane [3].

Several questions arise when we consider this problem :

'Dpto. de Matemáticas y Computación, Universidad de La Rioja, Logroño, Spain

(C) Australian Mathematical Society, 1994, Serial-fee code 0334-2700/94 
(i) The convergence in $\mathbb{C}$ of the infinite product $\prod_{n \geq 1}\left(1-z / w_{n}\right)$.

(ii) The existence of $D_{k}$ and convergence of the series $\sum_{k \geq 0}(-1)^{k} D_{k} z^{k}$.

(iii) Conditions on a bandlimited signal $f$ so that (1) can be satisfied.

The paper is organized as follows. In Section 1, (i) and (ii) are studied. We show that the second equality in (1) is valid if rank $\Lambda=m$ and $B_{1}, \ldots, B_{m}$ exist, where $B_{j}=\sum_{n \geq 1} w_{n}^{-j}$. In Section 2 we discuss the question (iii). Then, applying a result of Tichmarsh [4], we prove that a bandlimited signal $f$, normalized by $f(0)=1$, which has a symmetric spectrum with respect to the origin, can be written as

$$
f(z)=\prod_{n \geq 1}\left(1-\frac{z}{w_{n}}\right) \quad \text { where }\left|w_{1}\right| \leq\left|w_{2}\right| \leq \ldots \leq\left|w_{n}\right| \ldots
$$

Then, using the results obtained in Section 1, we prove that $f$ satisfies (1).

\section{Infinite products of Cardano type}

In what follows, $\Lambda=\left[w_{n}\right]_{n \geq 1}$ is an unbounded set of nonzero complex numbers, with no limit points and rank $m$; that is, $m$ is the smallest integer such that

$$
\sum_{n \geq 1}\left|w_{n}\right|^{-(m+1)}<+\infty
$$

DEFINITION. $\prod_{n \geq 1}\left(1-z / w_{n}\right)$ is said to be an infinite product of Cardano type if it coincides with $\sum_{k \geq 0}(-1)^{k} D_{k} z^{k}$ and defines an entire function, where

$$
D_{k}=\lim _{n} D_{k}^{(n)}, \quad \text { and } \quad D_{k}^{(n)}=\sum_{1 \leq i_{1} \leq \ldots \leq i_{k} \leq n} w_{i_{n}}^{-1} \ldots w_{i_{k}}^{-1} .
$$

We shall write $\Lambda \in C T$ when the associated product is of Cardano type.

This definition leads us firstly to study the existence of the $D_{k}$. We introduce the following notation: if $n \geq k$

$$
E_{i_{1}, \ldots, i_{k}}^{(n)}=\sum_{i_{i} \neq t_{j}, i \neq j} w_{i_{1}}^{-i_{1}} \ldots w_{t_{k}}^{-i_{k}}, \quad 1 \leq t_{j} \leq n, \quad i_{j} \in \mathbb{N} ; \quad B_{k}^{(n)}=\sum_{i=1}^{n} w_{t}^{-k} .
$$

When the limits exist, we write:

$$
E_{i_{1}, \ldots, i_{k}}=\lim _{n} E_{i_{1}, \ldots, i_{k}}^{(n)}
$$

and

$$
B_{k}=\lim _{n} B_{k}^{(n)}
$$

To simplify, we shall denote $E_{1,1,1,1,1}^{(n)}=E_{1, .(5 \ldots, 1}^{(n)}$, for example. 
THEOREM 1. $D_{k}$ exists for every $k$ if and only if $B_{k}$ exists for every $k$. Moreover

$$
D_{k+1}=\frac{(-1)^{k}}{k+1}\left[B_{k+1}+\sum_{t=1}^{k}(-1)^{t} D_{t} B_{k+1-t}\right] \text {. }
$$

PROOF. First we prove that if $n \geq k$ we have

$$
\begin{aligned}
E_{1, .(k \ldots, 1}^{(n)} B_{j}^{(n)} & =k E_{j+1,1,(k-1 \ldots, 1}^{(n)}+E_{j, 1, .(k ., 1}^{(n)}, \quad \text { for } j, k \in \mathbb{N} . \\
E_{j, 1, .(k \ldots, 1}^{(n)} & =(-1)^{k} k !\left[\sum_{t=0}^{k}(-1)^{t} D_{t}^{(n)} B_{k+j-t}^{(n)}\right], \quad \text { for } j, k \in \mathbb{N} . \\
E_{j, 1, .(k-j \ldots, 1}^{(n)} & =(-1)^{j-1}(k-j) !\left[k D_{k}^{(n)}+\sum_{t=1}^{j-1}(-1)^{t} D_{k-t}^{(n)} B_{t}^{(n)}\right], \\
& \text { for } 2 \leq j \leq k-1 \text { and } k \geq 3 .
\end{aligned}
$$

Indeed, when $n>k$ we obtain

$$
\begin{aligned}
E_{1_{, .(k \ldots, 1}}^{(n)} B_{j}^{(n)} & =\sum_{i=1}^{k}\left[\sum_{t_{p} \neq t_{q}, p \neq q} w_{t_{1}}^{-1} \ldots w_{t_{i}}^{-(j+1)} \ldots w_{t_{k}}^{-1}\right]+\sum_{t_{p} \neq t_{q}, p \neq q} w_{t_{1}}^{-1} \ldots w_{t_{k}}^{-1} w_{t_{k+1}}^{-j} \\
& =\sum_{i=1}^{k} E_{1, .(i-1, ., 1, j+1,1, .(k-i, \ldots, 1}^{(n)}+E_{1, .(k \ldots, 1, j}^{(n)} \\
& =k E_{j+1,1, .(k-1, \ldots, 1}^{(n)}+E_{j, 1,(k-1, \ldots 1}^{(n)}
\end{aligned}
$$

and it proves (6).

On the other hand, for $k=1$, (7) is deduced from (6) and from the equalities: $E_{1}^{(n)}=B_{1}^{(n)}=D_{1}^{(n)}$ and $E_{k}^{(n)}=B_{k}^{(n)}$. Now, from (6) we have

$$
\begin{aligned}
E_{j, 1, .(k \ldots 1}^{(n)} & =E_{1, .(k \ldots, 1}^{(n)} B_{j}^{(n)}-k E_{j+1,1,(k-1, \ldots, 1}^{(n)} \\
& =E_{1, .(k \ldots, 1}^{(n)} B_{j}^{(n)}-k\left[E_{1, .(k-1, ., 1}^{(n)} B_{j+1}^{(n)}-(k-1) E_{j+2,1, .(k-2 \ldots, 1}^{(n)}\right] .
\end{aligned}
$$

Then by using induction, (7) is proved by noting that $E_{1 .(k \ldots, 1}^{(n)}=k ! D_{k}^{(n)}$.

From (6), considering $k-1$ instead of $k$, we obtain

$$
E_{1,(k-1, \ldots 1}^{(n)} B_{1}^{(n)}=(k-1) E_{2,1, .(k-2 \ldots, 1}^{(n)}+E_{1, .(k, \ldots 1}^{(n)} .
$$

Then it follows that

$$
E_{2.1 . .(k-2 \ldots, 1}^{(n)}=(-1)(k-2) !\left[k D_{k}^{(n)}-D_{k-1}^{(n)} B_{1}^{(n)}\right] .
$$

Therefore (8) is verified for $j=2$. Now, (8) turns out to be immediate using induction on $j$.

As a consequence of this we have: 
(i) If $D_{1}, \ldots, D_{k}$ and $B_{j}, \ldots, B_{j+k}$ exist for any $j \geq 1$, then $E_{j, 1,(k \ldots, 1}$ exists for any $j \geq 1$.

(ii) If $D_{2}, \ldots, D_{k}$ and $B_{1}, \ldots, B_{k}$ exist with $k \geq 3$, then $E_{j, 1, .(k-j . .1}$ exists for $2 \leq j \leq k-1$.

Next we are going to prove the Theorem. Assume that $D_{m}$ exists for every $m$ and that $B_{2}, \ldots, B_{k}$ exist. Then from (6) $D_{1}^{(n)} B_{k}^{(n)}=B_{k+1}^{(n)}+E_{k, 1}^{(n)}$. Since $D_{1}, \ldots, D_{k+1}$ and $B_{1}, \ldots, B_{k-1}$ exist, it follows from (ii) that $E_{j, 1,(k+1-j \ldots, 1}$ exists for $2 \leq j \leq k$, and so $E_{k, 1}$ must exist. Now, taking limits in the last equality we obtain $B_{k+1}$.

Conversely, the existence of $B_{k}$ for any $k \in \mathbb{N}$ implies that $D_{1}$ exists. Using induction, (i) and the equality

$$
(k+1) ! D_{k+1}^{(n)}=k ! D_{k}^{(n)} B_{1}^{(n)}-k E_{2,1,(k-1, ., 1}^{(n)}
$$

obtained from (6), the existence of $D_{k+1}$ is proved by taking limits in this last expression. Finally, (5) is easily obtained by putting $j=1$ and taking limits in (7).

THEOREM 2. $\Lambda \in C T$ if and only if $B_{k}$ exists for $1 \leq k \leq m$.

PROOF. Since rank $\Lambda=m$, it is known [2], that the canonical product associated with $\Lambda$ converges uniformly on compact subsets (c.u.c.) of the plane, that is

$$
P_{m}(z)=\prod_{n \geq 1}\left(1-\frac{z}{w_{n}}\right) \exp \left[\sum_{j=1}^{m} \frac{z^{j}}{j w_{n}^{j}}\right] \text { c.u.c. }
$$

If $B_{1}, B_{2}, \ldots, B_{m}$ exist, it is not difficult to prove that

$$
\prod_{n \geq 1} \exp \left[-\sum_{j=1}^{m} \frac{z^{j}}{j w_{n}^{j}}\right]=\exp \left[-\sum_{j=1}^{m} B_{j} \frac{z^{j}}{j}\right],
$$

and therefore

$$
P(z)=\prod_{n \geq 1}\left(1-\frac{z}{w_{n}}\right) \text { c.u.c.. }
$$

Then

$$
\frac{P^{\prime}(z)}{P(z)}=\sum_{n \geq 1} \frac{1}{z-w_{n}} \text { c.u.c. in } D(0, R)=\{z:|z|<1\} \text {, }
$$

where $R<\left|w_{n}\right|$, for all $n$. If we let $g(z)=\sum_{n \geq 1} \frac{1}{z-w_{n}}$ then $g \in H(D(0, R))$ and $g^{(k)}(0)=(-1) k ! B_{k+1}$ for $k=0,1,2, \ldots$ As

$$
P^{(k+1)}(0)=\sum_{t=0}^{k}\left(\begin{array}{l}
k \\
t
\end{array}\right) P^{(t)}(0) g^{(k-t)}(0),
$$


it follows using induction that

$$
P^{(k+1)}(0)=(-1) k !\left[B_{k+1}+\sum_{t=0}^{k}(-1)^{t} D_{t} B_{k+1-t}\right] .
$$

Taking into account (5), we have that

$$
\prod_{n \geq 1}\left(1-\frac{z}{w_{n}}\right)=\sum_{k \geq 0}(-1)^{k} D_{k} z^{k}
$$

The converse is immediate.

Notice that in the case $m=0, \prod_{n \geq 1}\left(1-z / w_{n}\right)$ converges absolutely and c.u.c.. Moreover $\sum_{n \geq 1}\left|w_{n}\right|^{-k}$ exists for every $k$ and consequently the last result is trivial.

\section{Hadamard's factorization and the Taylor series for bandlimited signals}

A function $f$ on $\mathbb{R}$ is a bandlimited signal if its Fourier transform is zero outside a finite interval, i.e.

$$
\hat{f}(t)=\int_{-\infty}^{+\infty} f(z) e^{-i t z} d z=0 \text { for }|t|>\sigma
$$

and its energy,

$$
E=\int_{-\infty}^{+\infty}|f(z)|^{2} d z
$$

is finite.

It is easy to prove [3] that if $f$ is a bandlimited signal then $f$ is analytic in the entire plane and of exponential type. We obtain the Taylor series for a bandlimited signal $f$.

THEOREM 3. Let $f$ be a bandlimited signal, normalized by $f(0)=1$ and which has a symmetric spectrum with respect to the origin. Then

$$
f(z)=\sum_{k \geq 0}(-1)^{k} D_{k} z^{k} \quad \text { in } \mathbb{C} .
$$

PROOF. Let $F=f_{o} \phi$, where $\phi(z)=-i z$, and let $z_{1}=r_{1} e^{i \theta_{1}}, \ldots, z_{n}=r_{n} e^{i \theta_{n}}, \ldots$ be the zeros of $F$, arranged so that $r_{n}$ is a nondecreasing function of $n$. Then we can write

$$
F(z)=\frac{1}{2 \pi} \int_{-\sigma}^{\sigma} \hat{f}(t) e^{t z} d t
$$


where there is no number $\alpha>-\sigma$ such that $\int_{-\sigma}^{\sigma}|\hat{f}(t)| d t=0$ and no number $\beta<\sigma$ such that $\int_{\beta}^{\sigma}|\hat{f}(t)| d t=0$. It is known [4] that $F(z)=\prod_{n \geq 1}\left(1-z / z_{n}\right)$ where the product is conditionally convergent. Besides,

$$
\sum_{n \geq 1} \frac{\cos \theta_{n}}{r_{n}}=-\operatorname{Re}\left(\frac{F^{\prime}(0)}{F(0)}\right) \quad \text { and } \quad \sum_{n \geq 1} \frac{\sin \theta_{n}}{r_{n}}=-\operatorname{Im}\left(\frac{F^{\prime}(0)}{F(0)}\right) .
$$

From the above results, we have that $f(z)=\prod_{n \geq 1}\left(1-z / w_{n}\right)$, where $w_{n}=-i z_{n}$. Then, $\left[w_{n}\right]_{n \geq 1}$ is a rearrangement of the set $Z_{f}=\{z \in C \mid f(z)=0\}$.

To finish, we prove that $Z_{f} \in C T$. As

$$
B_{1}=\sum_{n \geq 1} \frac{1}{w_{n}}=\sum_{n \geq 1} \frac{\sin \theta_{n}}{r_{n}}+i \sum_{n \geq 1} \frac{\cos \theta_{n}}{r_{n}},
$$

necessarily $B_{1}$ exists. On the other hand, as $f$ is an entire function of exponential type, $B_{m}$ exists for all $m \geq 2[1]$. Therefore $Z_{f} \in C T$.

\section{Acknowledgement}

This research was supported by a grant of I. E. R.

\section{References}

[1] R. P. Boas, Entire functions (Academic Press, 1954).

[2] B. J. A. Levin, "Distribution of zeros of entire functions", Translations of Mathematical Monographs 5 (1980) 6-9.

[3] A. Papoulis, Signal analysis (McGraw Hill, 1986) 185-186.

[4] E. G. Titchmarsh, "The zeros of certain integral functions", Proc. London Math. Soc. 25 (2) (1926) 283-302. 